\title{
Legions of Presentations of Myxedema Coma: A Case Series from a Tertiary Hospital in India
}

\author{
Nirmalya Roy, ${ }_{1}^{1}$ Anirban Majumder, ${ }^{2}$ Debmalya Sanyal, ${ }^{2}$ Soumyabrata Roy Chaudhuri, ${ }^{2}$ \\ Suman Sarkar, ${ }^{1}$ Ankan Pathak ${ }^{1}$
}

${ }^{1}$ Department of Internal Medicine, KPC Medical College and Hospital, West Bengal University of Health Sciences, Kolkata, India
${ }^{2}$ Department of Endocrinology, KPC Medical College and Hospital, West Bengal University of Health Sciences, Kolkata, India

\begin{abstract}
Myxedema coma is associated with decreased mental status and hyponatremia among patients with diagnosed or undiagnosed hypothyroidism. The diagnosis is challenging in the absence of universally accepted diagnostic criteria, but should be considered as a differential even in cases with competing established diagnoses. All patients should receive intensive care level treatment. Even with optimal treatment, mortality is very high.
\end{abstract}

Key words: myxedema coma, presentations, outcomes, case reports

\section{INTRODUCTION}

Myxedema coma is a rare and potentially fatal condition. Even with the best possible treatment, mortality remains very high. ${ }^{1}$ Untreated hypothyroidism due to any cause including autoimmune disease, iodine deficiency, congenital abnormalities, drugs affecting thyroid function or secondary hypothyroidism can result in myxedema coma. The crisis is usually triggered by stressful events, the most common of which is infections. The incidence of myxedema coma in India is not known, but several case reports and case series have been published in recent years. $^{2-6}$ In developing countries recognition of this entity is made difficult by its slow onset, lack of awareness among both patients and physicians, and absence of diagnostic facilities in remote areas. Due to its rarity, physicians often fail to identify or keep it as a remote possibility while treating critically ill patients. This case series documents the myriad presentations of myxedema coma encountered in tertiary practice and encourages physicians to keep it in mind as a possibility while treating patients with altered sensorium.

\section{CASES}

\section{Case 1}

A 75-year-old female with a history of recurrent hospitalization for atrial fibrillation, heart failure, and sepsis was brought to the emergency room with circulatory collapse. She had been on amiodarone therapy for a long period of time, and was never diagnosed as having hypothyroidism. Investigation revealed hyponatremia and subnormal thyroid function. She was treated with conventional care including thyroid hormone and mechanical ventilation support but succumbed after a few days.

\section{Case 2}

A 70-year-old male with a long-standing history of hypothyroidism following radio-iodine treatment for Graves' disease was brought to the emergency room with progressively increasing sleepiness and altered sensorium. He did not reveal the history of radio-iodine therapy. Investigations showed community acquired pneumonia which was treated accordingly. Hyponatremia and subnormal thyroid function were detected later once the history of radio-iodine therapy was obtained. He recovered with thyroid hormone replacement and conventional care.

\section{Case 3}

A 75-year-old female with known hypothyroidism, type 2 diabetes, and hypertension presented in the emergency room with breathing difficulty and altered sensorium. She had hyponatremia and subnormal thyroid function. Investigations revealed the presence of heart failure with reduced ejection fraction. She was treated with standard care and with mechanical ventilatory support but succumbed after a few days.

\section{Case 4}

A 72-year-old female with known hypothyroidism presented in the emergency room with a history of bilateral lower limb swelling, facial puffiness and progressive unresponsiveness for four days. History from her attendants suggested that she had a very irregular intake of her thyroid medication. She was presumptively diagnosed as a case of myxedema coma and was treated with standard care before laboratory reports were made available. She eventually succumbed on the next day.
ISSN 0857-1074 (Print) I eISSN 2308-118x (Online)

Printed in the Philippines

Copyright (C) 2020 by Roy et al.

Received: June 22, 2020. Accepted: October 15, 2020.

Published online first: November 29, 2020.

https://doi.org/10.15605/jafes.035.02.12
Corresponding author: Prof. Anirban Majumder, MBBS

KPC Medical College and Hospital, West Bengal University of Health Sciences

1F Raja S.C. Mullick Road, Jadavpur, Kolkata, India 700032

Tel. No.: 033-6621 1700

Fax No.: 033-6621 1768

E-mail: dranirbanmazumdar@gmail.com

ORCiD: https://orcid.org//0000-001-6937-8675 


\section{Case 5}

A 68-year-old male, chronic alcoholic but not known to be hypothyroid, presented in the emergency room with a history of swelling of lower limbs and altered sensorium for two days. He was presumptively diagnosed as a case of hepatic encephalopathy and was treated accordingly. Investigations revealed hyponatremia and subnormal thyroid function. Myxoedema coma management was started three days after his hospitalization but he succumbed after a few days.

\section{Case 6}

An 83-year-old female, known hypertensive and hypothyroid, with a fractured femur operated one week earlier, became progressively drowsy over the past three days. Herpes zoster -related blisters on the left side of the neck was noted. Investigations revealed hyponatremia with subnormal thyroid function. She developed multiorgan failure and did not survive despite standard care and mechanical ventilatory support.

\section{Case 7}

A 50-year-old male, known hypothyroid and chronic alcoholic, was brought to the emergency room with inappropriate behavior. He was observed to be aggressive and impulsive. He was presumptively diagnosed and treated as a case of alcohol intoxication but did not improve. Investigations on the next day revealed hyponatremia, subnormal thyroid function, pericardial effusion and community acquired pneumonia. He recovered with conventional care.

\section{Case 8}

An 80-year-old female with known hypothyroidism, diabetes, and hypertension presented in the emergency room with abnormal mentation. She had a pale puffy face with very slow mentation. Investigations revealed hyponatremia, anemia, subnormal thyroid function with mildly elevated TSH and a huge pericardial effusion. She recovered with standard care for myxedema coma.

\section{Case 9}

A 92-year-old male, known to have hypertension, hypothyroidism, chronic kidney disease and with a permanent pacemaker, was admitted with massive fluid overload. After the fourth session of sustained lowefficiency dialysis (SLED), he became confused, agitated, and stuporous. Investigations revealed hyponatremia, subnormal thyroid function, and bilateral pleural effusion. He was treated with standard myxedema crisis care and with bi-level positive airway pressure support in the intensive care unit (ICU) and recovered in five days.

\section{Case 10}

A 66-year-old female with no current levothyroxine treatment was admitted to the ICU for myocardial infarction along with coma and bradycardia. Her core body temperature was documented at $88^{\circ} \mathrm{F}$. She had severe hyponatremia and urosepsis. She required mechanical ventilation but did not survive.

\section{Case 11}

A 71-year-old female not known to be hypothyroid was admitted to the ICU for myocardial infarction along with stupor and bradycardia. The core temperature was documented to be $90^{\circ} \mathrm{F}$. Aside from severe hyponatremia, she was also found to have urosepsis. She required mechanical ventilation but did not survive.

The case records of 11 patients with the diagnosis of myxedema coma between 1st January 2015 and 31st December 2019 admitted to our tertiary care hospital were reviewed (Table 1). Patients with poor clinical and/ or biochemical documentation were excluded from our study population. There are review articles and scoring systems to aid diagnosis, but these have limited sensitivity.? Myxedema coma is best recognized by the clinician when there is a high index of suspicion. The diagnosis in the case series was done clinically by the treating physicians. Data regarding age, sex, date of hospitalization, precipitating events, clinical presentation (central nervous system symptoms, heart rate, blood pressure, temperature), biochemical findings at presentation [free thyroxine (FT4), thyroid-stimulating hormone (TSH), random serum cortisol, serum sodium], management strategy (use of mechanical ventilation or noninvasive ventilation) and outcomes were retrieved from the documentation in the archival department of the hospital (Table 2).

Table 1. Presentations of myxedema coma cases

\begin{tabular}{|c|c|c|c|c|c|c|c|c|c|}
\hline Identifier & Age, yr & Sex & Season & Background & Precipitating event & $\mathrm{CNS}^{\mathrm{a}}$ symptoms & $H^{b}{ }^{b}$ & $\mathrm{BP}^{\mathrm{c}}$ & Temperature $\left({ }^{\circ} \mathrm{F}\right)$ \\
\hline Case 1 & 75 & $\mathrm{~F}$ & December & Not known hypothyroid & Amiodarone & Coma & 118 & $90 / 60$ & 98.4 \\
\hline Case 2 & 70 & M & October & Known hypothyroid & Pneumonia & Yes & 58 & $100 / 60$ & 98.7 \\
\hline Case 3 & 70 & $\mathrm{~F}$ & November & Known hypothyroid, T2DM ${ }^{d}$ and $H T N^{e}$ & Heart failure & Yes & 58 & $140 / 80$ & 97.2 \\
\hline Case 4 & 72 & $\mathrm{~F}$ & November & Known hypothyroid & Stopped LT4 ${ }^{f}$ & Yes & 60 & $100 / 60$ & 96.7 \\
\hline Case 5 & 68 & M & December & $\begin{array}{l}\text { Not known hypothyroid and known } \\
\text { alcoholic }\end{array}$ & $\begin{array}{l}\text { Hepatic } \\
\text { encephalopathy }\end{array}$ & Coma & 95 & $110 / 60$ & 95.9 \\
\hline Case 6 & 83 & $\mathrm{~F}$ & September & Known hypothyroid & Herpes zoster & Coma & 56 & $100 / 60$ & 96 \\
\hline Case 7 & 50 & M & December & Known hypothyroid and alcoholic & Pneumonia & Yes & 50 & $110 / 70$ & 97.4 \\
\hline Case 8 & 80 & $\mathrm{~F}$ & August & Known hypothyroid, T2DM ${ }^{d}$ and $\mathrm{HTN}^{\mathrm{e}}$ & Anaemia & Yes & 56 & $150 / 90$ & 97 \\
\hline Case 9 & 92 & M & November & Known hypothyroid, T2DM ${ }^{d}$ and $\mathrm{HTN}^{\mathrm{e}}$ & SLED ${ }^{g}$ & Yes & 78 & $160 / 80$ & 97 \\
\hline Case 10 & 66 & $\mathrm{~F}$ & November & Known hypothyroid and stopped LT4 & $\mathrm{MI}^{\mathrm{h}}$ & Coma & 58 & $90 / 60$ & 88 \\
\hline Case 11 & 71 & $\mathrm{~F}$ & October & Not known hypothyroid & Urosepsis & Yes & 52 & $80 / 58$ & 90 \\
\hline $\begin{array}{l}{ }^{\mathrm{a}} \mathrm{CNS} \text {, cent } \\
{ }^{\mathrm{b}} \mathrm{HR} \text {, heart } \\
{ }^{\mathrm{c}} \mathrm{BP}, \text { blood } \\
{ }^{\mathrm{d}} \mathrm{T} 2 \mathrm{DM} \text {, typ } \\
{ }^{\mathrm{e}} \mathrm{HTN} \text {, hype } \\
{ }^{\mathrm{f}} \mathrm{LT} 4 \text {, levoth } \\
{ }^{9} \mathrm{SLED} \text {, sus } \\
{ }^{\mathrm{h}} \mathrm{MI}, \text { myoca }\end{array}$ & $\begin{array}{l}\text { tral nervol } \\
\text { rate } \\
\text { pressure } \\
\text { e } 2 \text { diabe } \\
\text { ertension } \\
\text { hyroxine } \\
\text { stained lov } \\
\text { ardial infar }\end{array}$ & sy & ellitus & & & & & & \\
\hline
\end{tabular}


Table 2. Laboratory findings and outcome of myxedema coma cases

\begin{tabular}{|c|c|c|c|c|c|c|c|}
\hline Identifier & $\begin{array}{l}\text { FT4a, } \\
\text { ng/dL }\end{array}$ & $\begin{array}{l}\mathrm{TSH}^{\mathrm{b}} \\
\mathrm{mIU} / \mathrm{L}\end{array}$ & $\begin{array}{l}\text { Random serum } \\
\text { cortisol }^{c}, \mu g / d L\end{array}$ & $\begin{array}{c}\text { Serum Nad, } \\
\mathrm{mEq} / \mathrm{L}\end{array}$ & $\begin{array}{c}\text { Use of mechanical } \\
\text { ventilation }\end{array}$ & $\begin{array}{c}\text { Use of non-invasive } \\
\text { ventilation }\end{array}$ & Outcome \\
\hline Case 1 & 0.7 & 77.3 & Not done & 122 & Yes & No & Expired \\
\hline Case 2 & 0.56 & 37.7 & 12.7 & 117 & No & No & Recovered \\
\hline Case 3 & 0.7 & 77.32 & Not done & 133 & Yes & No & Expired \\
\hline Case 4 & 0.26 & $>100$ & Not done & 116 & No & No & Expired \\
\hline Case 5 & 0.26 & $>150$ & 12 & 124 & No & No & Expired \\
\hline Case 6 & 0.46 & 113 & 14 & 124 & Yes & No & Expired \\
\hline Case 7 & 0.36 & 37.7 & 10.7 & 105 & No & No & Recovered \\
\hline Case 8 & 0.6 & 10.3 & 9.7 & 120 & No & No & Recovered \\
\hline Case 9 & 0.24 & 31.22 & 17 & 122 & No & Yes & Recovered \\
\hline Case 10 & 0.3 & 124 & 21 & 119 & Yes & No & Expired \\
\hline Case 11 & 0.2 & 98 & 18 & 116 & Yes & No & Expired \\
\hline
\end{tabular}

Most of our patients were women (7 out of 11) and elderly (all above age 65 years except Case 7). Myxedema coma mostly develops in the winter months in patients with a history of thyroid disorders and a precipitating illness. ${ }^{1}$ Although Eastern India is not very cold during winter (average temperature of 12 to $26^{\circ} \mathrm{C}$ ), most of our patients presented early in the season (between September to December) and surprisingly not during the peak month (January). The presentation in India may be more common in winter months but can also occur at other times of the year. ${ }^{1}$ All but three (Cases 1, 5 and 11) had no previous history of thyroid disorders, posing a diagnostic challenge for the treating physicians. A significant number of patients with myxedema coma may not have had a previous history of thyroid disorders. ${ }^{1,2}$ Patients tend to forget their history of treatment for thyroid disorders (radio-iodine therapy or surgery) carried out many years earlier. This can lead to a delay in diagnosis and loss of precious time as illustrated in Case 2. All but one (Case 4) had a precipitating event. Sepsis or infection was the most common precipitating factor in our cohort as shown in other studies. ${ }^{2}$ Myxedema crisis may also be caused by discontinuation of thyroid supplementation as observed in Cases 4 and $10 .{ }^{1}$ The term myxedema coma is a misnomer as many patients present without coma. ${ }^{8}$ However, 4 out of 11 patients were hospitalized in comatose condition in our series, while the rest had altered mentation. As all the patients were elderly, dysglycemia, neurologic causes and sedative exposure were the primary considerations in those who presented with decreased sensorium. Appropriate history, laboratory and radiologic evaluation were done to rule out these common causes. It is noteworthy that not all patients presented with classic features of hypothermia, bradycardia and hypotension. ${ }^{8}$ The most common findings were a combination of altered mental status and hyponatremia. Hypothermia (temperature below $97^{\circ} \mathrm{F}$ ) was observed only in five patients and seen only with rectal temperature measurement. The incidence of severe hypothermia is expected to be low in India. ${ }^{1}$ Considering the variety of presentations, physicians must have a high index of suspicion in all cases presenting with altered mentation. Even in patients with competing established diagnoses, such as encephalopathy from alcoholic chronic liver disease, the possibility of myxedema crisis should still be considered, as found in Case 5 of our cohort.

\section{DISCUSSION}

The diagnosis of myxedema coma is based on history (especially with an identified precipitating event), physical findings (specifically hypothermia, hypotension, bradycardia, and hypoventilation), deteriorating mental status and laboratory abnormalities. ${ }^{8}$ No single diagnostic test can confirm or exclude the diagnosis. In suspected cases, a random blood sample should be drawn prior to treatment for the measurement of TSH, FT4 and serum cortisol. Laboratory results showed low FT4 and elevated TSH in all the cases. The TSH value was not significantly high in one case (Case 8), and the low FT4 value raises the possibility of secondary hypothyroidism.

Myxedema coma is the final stage of severe long-standing hypothyroidism, associated with marked impairment of central nervous system function, cardiovascular decompensation and high mortality rate, mostly seen in the elderly during the winter months. ${ }^{9}$ Clinically there is subnormal temperature as low as $23^{\circ} \mathrm{C}$, bradycardia, hypotension, delay in deep tendon reflexes, seizures and coma. In the background of untreated hypothyroidism, myxedema coma is induced by exposure to cold environments, surgery, trauma, cerebrovascular accidents, gastrointestinal bleeding, heart failure, infections like pneumonia or urosepsis, but the usual signs of infection (fever, diaphoresis, tachycardia) are generally absent., ${ }^{1,-11}$ Medications like anesthetics, sedatives, narcotics, lithium, amiodarone, sunitinib and phenytoin can precipitate myxedema coma. ${ }^{1}$ Thyroid hormone activates mitochondrial metabolism, stimulates nuclear receptors through cell membrane $\mathrm{Na}^{+}, \mathrm{K}^{+}$-ATPase and increases oxygen consumption leading to a characteristic increase in basal metabolic rate. ${ }^{9}$ Severe hypothermia (core temperature less than $90^{\circ} \mathrm{F}$ or $32.2^{\circ} \mathrm{C}$ ), hyponatremia, decreased cerebral blood flow, hypoxemia and sepsis can lead to altered mental status with lowering of seizure threshold in myxedema. ${ }^{1,10}$ Altered respiratory sensitivity to hypoxia and hypercapnia, reduction in respiratory drive, pneumonia, along with respiratory muscle dysfunction, can lead to hypoventilation. ${ }^{1,11,12}$ In addition, myxedematous swelling of the upper airway with macroglossia, pleural effusion and obstructive sleep apnea can further aggravate hypoxia and carbon dioxide retention. ${ }^{11}$ 


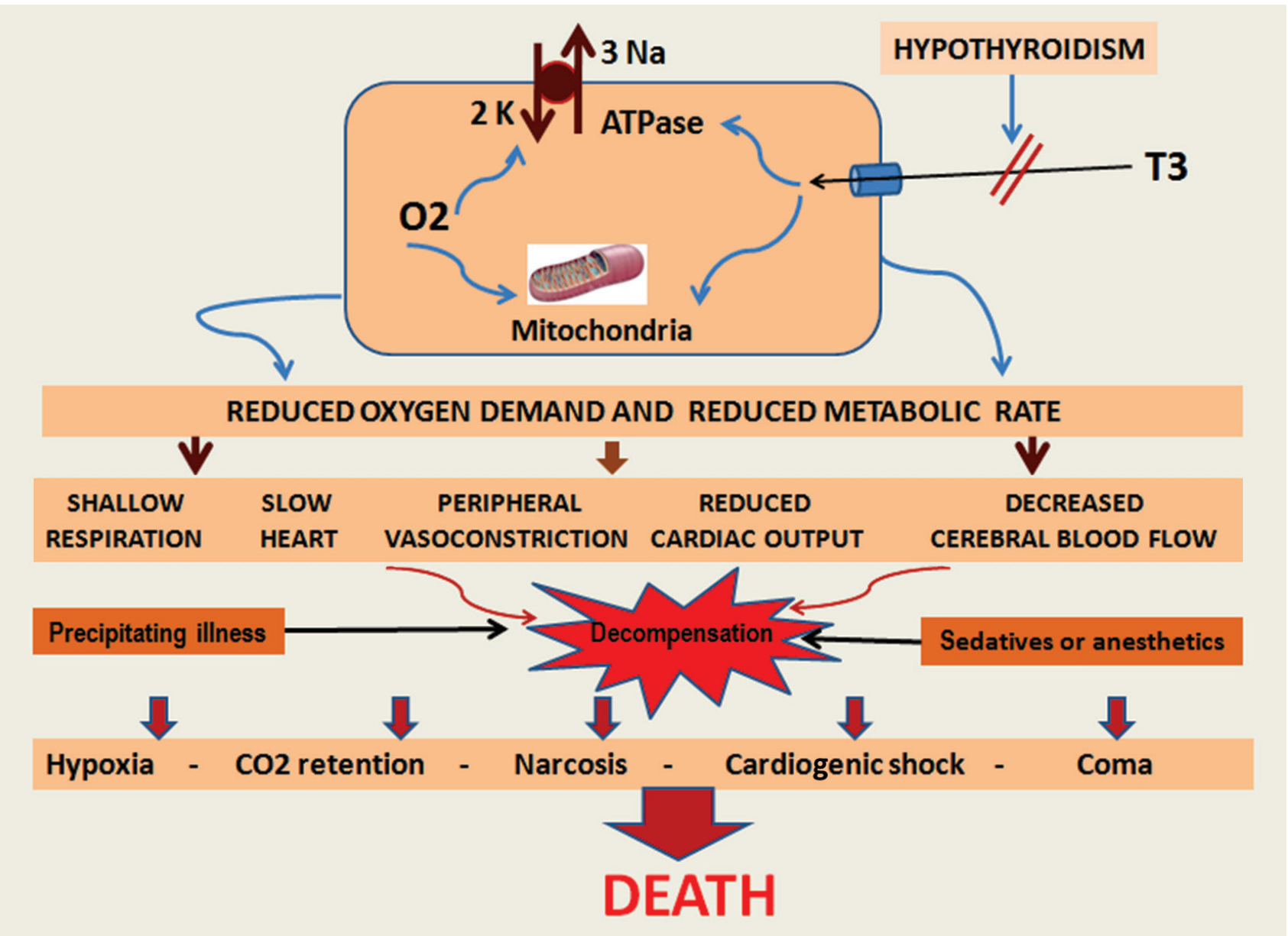

Figure 1. Pathophysiology of myxoedema coma.

Negative inotropic and chronotropic alterations in hypothyroidism, manifested as decreased stroke volume, bradycardia and decreased cardiac output, precipitate cardiogenic shock. ${ }^{1,10,13}$ Increase in $\alpha$-adrenergic responsiveness in hypothyroidism causes peripheral vasoconstriction, which shunts blood away from skin and muscle to maintain core body temperature and presents with the characteristic finding of cool and pale skin. In addition, accumulation of mucopolysaccharides and water can result in pericardial effusion obscuring ischemic findings but may also result in cardiac tamponade physiology. ${ }^{14}$ In response to vasoconstriction, there is a reduction in blood volume by as much as $20 \%$, while a reduction in erythropoietin levels lead to a decline in red cell production and fall in hematocrit by approximately 30\%. Hyponatremia occurs due to diminished capacity to clear free water load as a consequence of the combined effects of lower renal perfusion and inappropriately elevated antidiuretic hormone levels despite low serum osmolality. ${ }^{10,11,15}$ Increased insulin sensitivity, poor appetite and potential simultaneous adrenal insufficiency impairing gluconeogenesis, all contribute to hypoglycemia in severe hypothyroidism. ${ }^{1,15}$ Reduced intestinal motility in severe hypothyroidism may reduce absorptive efficiency contributing to paralytic ileus with abdominal distention. ${ }^{10}$ Further sluggish circulation and severe hypometabolism impair absorption of therapeutic agents from the gut or from subcutaneous or intramuscular sites. As such, medications should be administered intravenously if possible.
Dose, preparation and route of administration of levothyroxine (LT4) have always been a matter of debate. In some institutions, intravenous thyroxine (T4) or a combination of triiodothyronine (T3) and T4 are used. While oral T3 is not available in India, oral T4 is easily available. However, administration of T4 through Ryles tube is equally effective as intravenous T4, with the advantage of easier interpretation of serum T4. ${ }^{1,5}$ Despite following a standard protocol for myxedema management (empiric antibiotic, dextrose-saline infusion, thyroxine sodium 300 to $500 \mu \mathrm{g}$ through Ryles tube, intravenous hydrocortisone $100 \mathrm{mg}$ every 8 hours, warming blanket to prevent heat loss and ventilatory support if required), seven out of 11 expired in our institute. No adverse event, especially cardiac, was documented with such a high dose of thyroxine sodium. Patients with hypoventilation (six out of 11) required ventilatory support; most of them (five out of 6) expired. Predicting the outcome of the patients with myxedema coma is difficult. However, hypotension and bradycardia at presentation, need for mechanical ventilation, unresponsive hypothermia, presence of sepsis, intake of sedative drugs, low Glasgow Coma Scale and high APACHE II score are proposed as possible predictors for mortality. ${ }^{5}$

Our study has several limitations. First, recorded diagnoses in retrospective real-world studies are less well-validated than those in well-planned randomized controlled trials. Hence, the generalizability of our results may be limited. Second, our results were mainly based 
on enteral administration of levothyroxine; intravenous thyroxine remains as the standard therapy for patients with myxedema coma. Third, post-discharge mortality information is not available to us. Lastly, we could not perform multivariate logistic regression analysis of all potential risk factors for myxedema coma mortality because of the small sample size.

\section{CONCLUSION}

In the absence of a definitive diagnostic tool, myxedema coma is largely a clinical diagnosis. In view of the myriad of presentations and absence of classic features in many situations, a high index of suspicion is required for a timely diagnosis. In elderly people presenting with hyponatremia and decreased sensorium, myxedema coma should be considered as a differential diagnosis. Despite standard treatment after detection, myxedema coma is associated with poor outcomes.

\section{Ethical Consideration}

Patients' consent were obtained before submission of the manuscript.

\section{Statement of Authorship}

All authors certified fulfillment of ICMJE authorship criteria.

\section{Author Disclosure}

All authors declared no conflicts of interest.

\section{Funding Source}

None.

\section{References}

1. Mathew V, Misgar RA, Ghosh S, et al. Myxedema coma: A new look into an old crisis. J Thyroid Res. 2011;2011:493462. PMID: 21941682. PMCID: PMC3175396. https://doi.org/10.4061/2011/493462.

2. Murthy TA, Rangappa P, Jacob IPR, Janakiraman R, Rao K. Myxoedema coma in adults: Experience from a tertiary referral hospital intensive care unit. Indian J Anaesth. 2015;59(5):315-7. PMID: 26019358 PMCID: PMC4445155. https://doi.org/10.4103/0019-5049.156889.
3. Mylliemngap B, Swain S, Vyas S, Kumar P. Myxedema coma, pancytopenia, and hypocoagulopathy: A rare presentation of Sheehan's syndrome. Indian J Endocrinol Metab. 2019;23(2):268-9. PMID: 31161117. PMCID: PMC6540897. https://doi.org/10.4103/ijem. IJEM_120_19.

4. Baduni N, Sinha SK, Sanwal MK. Perioperative management of a patient with myxedema coma and septicemic shock. Indian J Crit Care Med. 2012;16(4):228-30. PMID: 23559735. PMCID: PMC3610460. https://doi.org/10.4103/0972-5229.106510.

5. Dutta P, Bhansali A, Masoodi SR, Bhadada S, Sharma N, Rajput R. Predictors of outcome in myxoedema coma: A study from a tertiary care centre. Crit Care. 2008:12(1):R1. PMID: 18173846. PMCID: PMC2374608. https://doi.org/10.1186/cc6211.

6. Ono Y, Ono S, Yasunaga H, Matsui H, Fushimi K, Tanaka Y. Clinical characteristics and outcomes of myxedema coma: Analysis of a national inpatient database in Japan. J Epidemiol. 2017;27(3):117-22. PMID: 28142035. PMCID: PMC5350620. https://doi.org/10.1016/j.je. 2016.04.002.

7. Popoveniuc G, Chandra T, Sud A, et al. A diagnostic scoring system for myxedema coma. Endocr Pract. 2014;20(8):808-17. PMID: 24518183. https://doi.org/10.4158/EP13460.OR.

8. Wall CR. Myxedema coma: Diagnosis and treatment. Am Fam Physician. 2000;62(11):2485-90. PMID: 11130234

9. Nicoloff JT, LoPresti JS. Myxedema coma. A form of decompensated hypothyroidism. Endocrinol Metab Clin North Am. 1993;22(2):279-90. PMID: 8325287.

10. Rodrigo C, Gamakaranage CS, Epa DS, Gnanathasan A, Rajapakse S. Hypothyroidism causing paralytic ileus and acute kidney injury - case report. Thyroid Res. 2011:4(1):7. PMID: 21303532. PMCID: PMC3041782. https://doi.org/10.1186/1756-6614-4-7.

11. Abuzaid AS, Birch N. The controversies of hyponatraemia in hypothyroidism: Weighing the evidence. Sultan Qaboos Univ Med J. 2015;15(2):e207-12. PMID: 26052453. PMCID: PMC4450783.

12. Zwillich CW, Pierson DJ, Hofeldt FD, Lufkin EG, Weil JV. Ventilatory control in myxedema and hypothyroidism. N Engl Med. 1975;292(13):662-5. PMID: 1113761. https://doi.org/10.1056/ NEJM197503272921302.

13. Silva EI, Landsberg L. "Catecholamines and the sympathoadrenal system in hypothyroidism." In: The Thyroid. LE Braverman, RD Utiger, eds. Philadelphia: J.B. Lippincott Co., 1992.

14. Schenck JB, Rizvi AA, Lin T. Severe primary hypothyroidism manifesting with torsades de pointes. Am J Med Sci. 2006:331(3):1546. PMID: 16538077. https://doi.org/10.1097/00000441-200603000-00008.

15. Iwasaki Y, Oiso Y, Yamauchi K, et al. Osmoregulation of plasma vasopressin in myxedema. J Clin Endocrinol Metab. 1990;70(2):534-9. PMID: 2298864. https://doi.org/10.1210/jcem-70-2-534.

Authors are required to accomplish, sign and submit scanned copies of the JAFES Author Form consisting of: (1) Authorship Certification, that authors contributed substantially to the work, that the manuscript has been read and approved by all authors, and that the requirements for authorship have been met by each author; (2) the Author Declaration, that the article represents original material that is not being considered for publication or has not been published or accepted for publication elsewhere, that the article does not infringe or violate any copyrights or intellectual property rights, and that no references have been made to predatory suspected predatory journals; (3) the Author Contribution Disclosure, which lists the specific contributions of authors; and (4) the Author Publishing Agreement which retains author copyright, grants publishing and distribution rights to JAFES, and allows JAFES to apply and enforce an Attribution-Non-Commercial Creative Commons user license. Authors are also required to accomplish, sign, and submit the signed ICMJE form for Disclosure of Potential Conflicts of Interest. For original articles, authors are required to submit a scanned copy of the Ethics Review Approval of their research as well as registration in trial registries as appropriate. For manuscripts reporting data from studies involving animals, authors are required to submit a scanned copy of the Institutional Animal Care and Use Committee approval. For Case Reports or Series, and Images in Endocrinology, consent forms, are required for the publication of information about patients; otherwise, appropriate ethical clearance has been obtained from the institutional review board. Articles and any other material published in the JAFES represent the work of the author(s) and should not be construed to reflect the opinions of the Editors or the Publisher. 